\title{
Extra! Extra! Read all about it!
}

The idea that centrosome aberrations could drive tumour development was proposed more than 100 years ago and yet there is still uncertainty as to whether extra centrosomes are a cause or a consequence of tumorigenesis in mammals. Furthermore, the mechanisms accounting for the faithful control of centrosome number are not fully understood. Two papers now demonstrate the oncogenic potential of centrosome amplification in mammals and describe the pathway that acts to halt cell proliferation in response to extra centrosomes.

The acquisition of super-

extra

centrosomes

were sufficient

to initiate tumorigenesis in mammals

5
numerary centrosomes is frequently observed in the early development of tumours, but studies in animal models have so far been contradictory. Levine et al. addressed this controversy by developing a mouse model in which the overexpression of Polo-like kinase 4 (PLK4), a crucial regulator of centrosome duplication, could be induced by doxycycline ( $\left.P l k 4^{\mathrm{DOX}}\right)$ to pro-

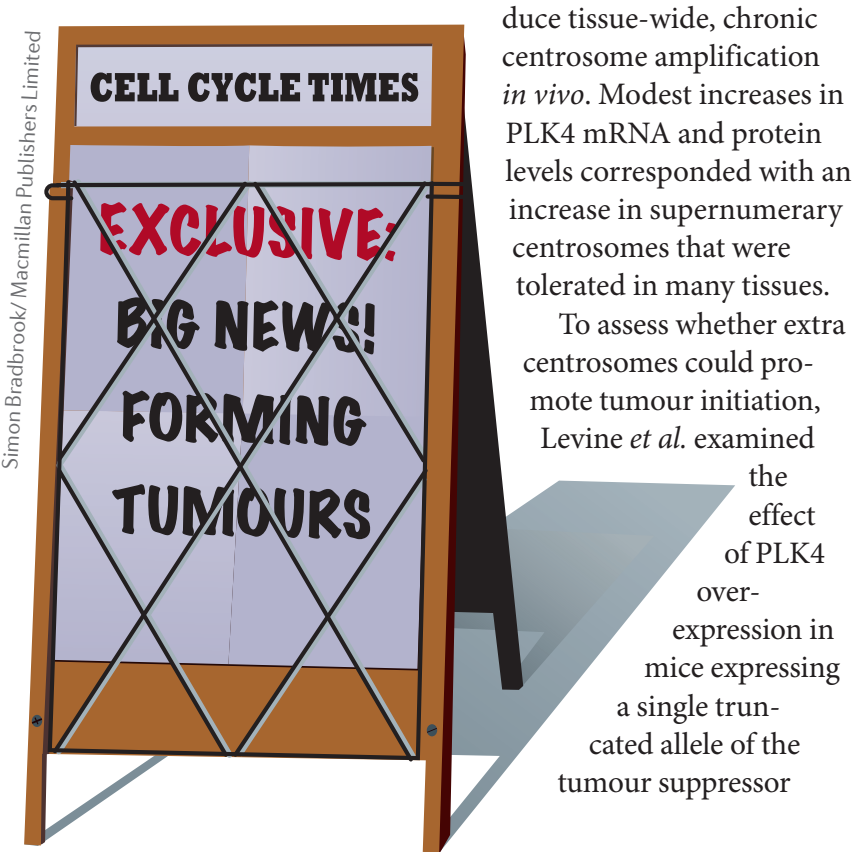

adenomatous polyposis coli $(A p c)-\mathrm{a}$ mouse model of intestinal neoplasia $\left(A p c^{\mathrm{MIN} /+}\right) . A p c^{\mathrm{MIN} /+} ; P l k 4^{\mathrm{DOX}}$ mice developed significantly more tumours than $A p c^{\mathrm{MIN} /+}$ mice, consistent with an increased centrosome number observed in both the normal intestine and in the intestinal tumours. However, as tumour size was not different between the two mouse populations, the authors concluded that centrosome amplification drives the initiation but not the progression of intestinal tumours. Next, to test whether extra centrosomes were sufficient to initiate tumorigenesis in mammals, age-matched $P l k 4^{\text {DOX }}$ and control mice were fed doxycycline from 1 to 2 months of age. By 36 weeks, $P l k 4^{\text {Dox }}$ mice had developed spontaneous tumours - lymphomas, squamous cell carcinomas and sarcomas - all of which displayed high levels of centrosome amplification, aneuploidy and karyotypic diversity. Most tumour cells were found to contain only one or two extra centrosomes, which importantly, is comparable to the extent of centrosome amplification in most human tumours.

A common finding in all previous studies of centrosome amplification has been that tissues that retain functional p53 do not tolerate extra centrosomes and undergo a p53-dependent proliferative arrest to maintain genomic integrity. Fava et al. investigated the molecular pathway by which p53 becomes activated upon centrosome amplification in cancer cell lines. The authors disrupted cytokinesis using Aurora B kinase inhibition in A549 lung adenocarcinoma cells or forced the generation of extra centrosomes through overexpression of PLK4 in U2OS osteosarcoma cells. These conditions resulted in the cleavage of
MDM2 (a negative regulator of p53) through the activity of caspase 2 , and the subsequent upregulation of p53 and p21-dependent cell cycle arrest.

Given that caspases are activated in multicomponent protein complexes, the authors reasoned that caspase 2 activation upon cytokinesis failure or in the presence of extra centrosomes might be through the molecular platform comprising caspase 2, p53-induced death domain-containing protein 1 (PIDD1) and receptor-interacting protein (RIP)-associated protein with a death domain (RAIDD), collectively known as the PIDDosome. In accordance with this, CRISPRCas9-mediated deletion of each individual PIDDosome component disrupted MDM2 cleavage and p53 accumulation, allowing proliferation to proceed and suggesting that the PIDDosome has an unexpected pro-survival function upstream of p53 in detecting centrosome number. Interestingly, Levine et al. found that the thymic lymphomas developing in Plk4 ${ }^{\text {DOX }}$ mice had decreased levels of p53 target genes, implying that the p53 pathway is at least partially compromised in spontaneous tumours that arise following centrosome amplification.

Together, these two studies propose that extra centrosomes can be causal in mammalian cancers and indicate that the $\mathrm{p} 53$ pathway, which includes the PIDDosome, acts as an initial barrier to the continued proliferation of cells with extra centrosomes.

Anna Dart

ORIGINAL ARTICLES Levine, M. S. et al. Centrosome amplification is sufficient to promote spontaneous tumorigenesis in mammals. Dev. Cell http://dx.doi.org/10.1016/j.devcel.2016.12.022 (2017) | Fava, L. L. et al. The PIDDosome activates p53 in response to supernumerary centrosomes. Genes Dev. 31, 34-45 (2017) 